Eduardo Rosas Rojas"

Teresa López González"

Universidad Autónoma del Estado de México Atizapán de Zaragoza, Estado de México

Recibido: 5 de noviembre de 2017 Concepto de evaluación: 27 de junio de 2018 Aprobado: 27 de junio de 2018

Artículo de investigación (C) 2018 Universidad Católica de Colombia. Facultad de Ciencias Económicas y Administrativas. Todos los derechos reservados

* Doctor en Economía, Profesorinvestigador, de la Universidad Autónoma del Estado de México (UAEM), Ciudad de México, México. Miembro del Sistema Nacional de Investigadores del CONACYT". Correo electrónico: erosasr@uaemex.mx.http:// orcid.org/0000-0002-7255-7778.

** Doctorado en Economía, Profesora de la Universidad Nacional Autónoma de México (UNAM), Ciudad de México, México; miembro del Sistema Nacional de Investigadores del CONACYT. Correo electrónico: tere_slg@unam.mx.https:// orcid.org/0000-0002-8124-2292.
Finanz. polit. econ., ISSN: 2248-6046, Vol. 10, N. ${ }^{\circ} 2$, julio-diciembre, 2018, pp. 349-372

http://dx.doi.org/10.14718/revfinanzpolitecon.2018.10.2.6

\section{Inflación e incertidumbre inflacionaria: la postura del Banco de México, 1969-2017*}

\section{RESUMEN}

Este artículo examina la relación entre inflación e incertidumbre inflacionaria para la economía de México durante el periodo que comprende enero de 1969 a febrero de 2017, utilizando modelos SARMA-GARCH y sus extensiones GJR-GARCH-M y E-GARCH-M. Entre los hallazgos es posible evidenciar un efecto causal negativo de la incertidumbre inflacionaria sobre la inflación (cumplimiento de la hipótesis de Holland), lo cual indica que el Banco de México exhibe un comportamiento estabilizador ante la inflación. También se corrobora el cumplimiento de la hipótesis de Friedman-Ball, que establece que altos niveles de inflación incrementan la incertidumbre inflacionaria.

Palabras clave: Banco Central, efectos asimétricos, incertidumbre inflacionaria, inflación.

JEL: C51, C55, E31

\section{Inflation and inflation uncertainty: The position of the Bank of Mexico, 1969-2017}

\section{ABSTRACT}

This research paper examines the relationship between inflation and inflation uncertainty for the Mexican economy during the period from January 1969 to February 2017. Using SARMA-GARCH models and their extensions (GJR-GARCH-M and E-GARCH-M), the study evidenced a negative causal effect of financial uncertainty on inflation (compliance with Holland's hypothesis), which indicates that the Bank of Mexico exhibits a stabilizing behavior in relation to inflation. It also confirms the fulfillment of the Friedman-Ball hypothesis, which states that high levels of inflation increase inflation uncertainty.

Keywords: asymmetric effects, Central Bank, inflation, inflation uncertainty.

El artículo es resultado de la investigación de los autores, registrada como parte de la colaboración conjunta en el nombre del Cuerpo Académico en Economía Financiera del Centro Universitario UAEM Valle de México y la Facultad de Estudios Superiores Acatlán, UNAM 


\section{Inflação e incerteza inflacionária: o posicionamento do Banco do México, 1969-2017}

\section{RESUMO}

Este artigo examina a relação entre inflação e incerteza inflacionária para a economia do México durante o período que compreende de janeiro de 1969 a fevereiro de 2017, utilizando modelos SARMA-GARCH e suas extensões GJR-GARCH-M e E-GARCH-M. Entre os achados, é possível evidenciar um efeito causal negativo da incerteza inflacionária sobre a inflação (comprimento da hipótese de Holland), o que indica que o Banco do México apresenta um comportamento estabilizador ante a inflação. Também se corrobora o cumprimento da hipótese de Friedman-Ball, que estabelece que altos níveis de inflação aumentam a incerteza inflacionária.

Palavras-chave: Banco Central, efeitos assimétricos, incerteza inflacionária, inflação. 


\section{INTRODUCCIÓN}

Los episodios de elevada inflación en México durante las décadas de 1970 y 1980, que alcanzó tres dígitos, configuró el consenso de que la autoridad monetaria debía enfocarse principalmente en alcanzar y mantener un entorno de inflación baja y estable. Las políticas de estabilización y ajuste macroeconómico implementadas entre 1983 y 1987 fracasaron en ese objetivo de corto plazo; será hasta 1989 cuando se logre una reducción drástica y acelerada de la tasa de inflación anual, la cual pasó de $159,2 \%$ en 1987 a $51,5 \%$ en 1988 , y a $19,7 \%$ en 1989 , como resultado de la política de ingreso implementada en los primeros años de la administración del presidente Salinas de Gortari (Sidaoui, 2008, p. 5).

El comportamiento de la tasa de inflación durante el periodo 1990-1994 muestra los esfuerzos de la autoridad monetaria por controlar la tasa de inflación, mediante el sistema de bandas cambiarias previamente fijadas para evitar que las fluctuaciones en el tipo de cambio se trasladaran al nivel de precios (Mántey, 2010). Se suponía que el otorgamiento de la autonomía al Banco de México en 1994 contribuiría a consolidar la estabilidad monetaria, pues bajo ese nuevo marco normativo el Instituto asumía la estabilidad de precios como objetivo prioritario de la política monetaria; sin embargo, la devaluación del peso por parte de la autoridad monetaria para hacer frente a la fuga de capitales previa precipitó la crisis cambiaria-financiera a finales de 1994, con la consecuente reversión repentina de los flujos de capital de cartera y el incremento de la tasa de interés. Las presiones inflacionarias resurgieron como consecuencia del significativo ajuste cambiario, y en los años posteriores para afrontar esta crisis se instrumentaron medidas monetarias restrictivas: la contracción del crédito interno neto, por un lado, y una política de intervención en el mercado cambiario, por el otro, con el propósito de esterilizar los efectos monetarios que generan los flujos de capital de cartera en la base monetaria. Esta estrategia implica una elevada acumulación de reservas internacionales.
A partir de 1998, una vez se logró la estabilidad del sistema de pagos, la política monetaria se orientó al logro de tasas de inflación bajas; para ello, se adoptó una estrategia basada en los siguientes objetivos: a) mantener el "corto" con el propósito de reducir la inflación de manera sostenible; b) responder de forma adecuada a los choques inflacionarios; c) mejorar la transparencia en la conducción de la política monetaria (Ramos-Francia y Torres, 2005).

En 1999 era evidente el proceso de convergencia gradual hacia un esquema de objetivos de inflación, ya que el Banco de México anunció por primera vez una meta de mediano plazo para la inflación. A principios de 2001 se adopta formalmente dicho esquema, al establecer la meta de inflación en una tasa de $3 \pm 1 \%$. La variación de $1 \%$ responde a la incidencia que puedan tener los factores de corto plazo sobre el comportamiento de dicha tasa; en otras palabras, este margen de variación en la meta busca representar de forma práctica la incertidumbre asociada al comportamiento de la inflación.

En general, es posible decir que desde 2003 el esquema de metas de inflación ha sido exitoso en el cumplimiento de la meta fijada, pues las expectativas de inflación se han estabilizado en torno a la meta planteada por el Banco de México, mientras que la volatilidad de las expectativas ha decrecido como consecuencia de un entorno inflacionario estable y un ambiente de certidumbre. Lo anterior ha significado una contracción en el crecimiento del producto (Ball, 1992).

La comprensión del movimiento de las expectativas sobre la evolución de los precios es importante para mantener la estabilidad del poder adquisitivo del peso mexicano, pues estas inciden en la inflación observada. Lo anterior se desarrolla de esta forma por cuanto las modificaciones en precios que llevan a cabo los agentes económicos dependen, en parte, de la inflación que, intuyen, se presentará en el futuro; por tanto, la credibilidad del Instituto Central sobre su compromiso con la estabilidad de la inflación es crucial para que el Banco de México pueda mantener un entorno de inflación moderada y estable (Aguilar-Argaez et al., 2014). 
El presente artículo de investigación analiza la relación entre la inflación y la incertidumbre inflacionaria entre 1969 y 2017. Se ha demostrado que la incertidumbre asociada al comportamiento de la inflación conduce a la mala asignación de recursos y genera ineficiencias en la economía. Por sus fluctuaciones drásticas en el comportamiento de la inflación, esto es, periodos de elevada inflación seguidos de periodos de estabilización monetaria, la economía mexicana representa un caso importante para el análisis empírico de la naturaleza de la relación entre la inflación y la incertidumbre inflacionaria; concretamente, se analiza si existe una retroalimentación mutua de estas dos variables a lo largo de las últimas seis décadas. La hipótesis que guía la investigación sostiene que si la incertidumbre nominal se incrementa en la medida que lo hace la inflación del periodo previo, la autoridad monetaria habrá adoptado una postura antiinflacionaria para disminuir la incertidumbre sobre la inflación futura.

La estructura del artículo se compone de cuatro secciones. En la segunda se presenta una revisión de la literatura relevante sobre el nexo existente entre la inflación y la incertidumbre inflacionaria; la tercera presenta la metodología econométrica de los modelos autorregresivos y de medias móviles estacionales (SARMA, por sus siglas en inglés) y los modelos de heteroscedasticidad condicional autorregresiva generalizada (GARCH, por sus siglas en inglés) y sus extensiones GJR-GARCH-M y E-GARCH-M; en la cuarta sección se presenta la estimación de los modelos y los resultados empíricos más destacados, y finalmente se exponen las principales conclusiones derivadas de la evidencia empírica obtenida.

\section{REVISIÓN DE LA LITERATURA}

Uno de los principales costos que genera la inflación es la incertidumbre respecto a la evolución de los precios en el futuro, ya que, por un lado, provoca distorsiones en la toma de decisiones de consumidores y empresas y, por otro, reduce el bienestar económico debido a la reducción de valor de los ingresos reales. En ausencia de incertidumbre o en un contexto de estabilidad de precios, los agentes económicos podrían mejorar la planeación futura (Golob, 1994, p. 27). La incertidumbre inflacionaria futura puede afectar la toma de decisiones de inversión, ahorro y consumo, lo cual tiene consecuencias económicas adversas que potencialmente aumentarán con la inflación.

La incertidumbre sobre la evolución futura de la inflación presenta dos tipos de consecuencias económicas: en primer lugar, provoca que las empresas y los consumidores tomen decisiones económicas que difieren de aquellas que habrían adoptado en un contexto de certidumbre. A esto se le conoce como efecto ex ante, ya que las decisiones prevén el futuro incremento de precios; el segundo efecto se presenta después de que las decisiones han sido tomadas, es decir, son efectos ex post, los cuales tienen lugar cuando el incremento en los precios difiere de lo que se había esperado.

Si bien las consecuencias de la incertidumbre inflacionaria son fácilmente identificables, es más complejo explicar por qué esta se incrementa con la inflación. La explicación más llamativa se relaciona con una respuesta de política monetaria ante la inflación, pues cuando la inflación es baja, los responsables de dicha política tratan de mantenerla baja. Así, en la medida en que la política monetaria sea exitosa en el control de la inflación, es muy probable que la inflación se mantenga estable y en bajos niveles. Sin embargo, cuando se presenta un incremento en los precios, los responsables de la política monetaria son más propensos a adoptar políticas antiinflacionarias; pero estas políticas, en su esfuerzo por reducir la tasa de inflación, elevan la variabilidad inflacionaria (Golob, 1994, p. 28).

Friedman (1977) establece un argumento informal de dos partes sobre los efectos reales de la inflación: el primero sostiene que un incremento en la inflación puede inducir una respuesta de política errática por parte de la autoridad monetaria y, por lo tanto, generar más incertidumbre sobre la tasa de inflación futura (la llamada hipótesis de Friedman); en el segundo argumento se asume que la incertidumbre inflacionaria tiene un efecto negativo sobre el crecimiento económico. 
Ambas afirmaciones informales fueron precisadas algebraicamente por Ball (1992), que presenta un modelo de juego repetido del tipo Barro-Gordon con información asimétrica, donde el público enfrenta la incertidumbre sobre el tipo de funcionario público en el cargo. Este político alterna estocásticamente en el puesto y puede ser de dos tipos: un funcionario débil, incapaz de aplicar políticas antiinflacionarias, y otro funcionario fuerte preparado para soportar el costo de estas políticas. Durante los periodos de alta inflación, la incertidumbre sobre el tipo de político que estará en el cargo en los próximos periodos incrementará la incertidumbre sobre la tasa de crecimiento del dinero y, en consecuencia, también sobre la tasa de inflación futura. En estos esquemas se asevera que, como resultado de incrementos en los precios, hay una mayor volatilidad inflacionaria en el futuro, por lo que se presenta una correlación positiva entre ambas; así también que la incertidumbre inflacionaria depende de la inflación rezagada (Rocabado, 2009).

Recientemente, se ha desarrollado un creciente interés en comprobar la relación empírica entre inflación e incertidumbre inflacionaria; esta última usualmente se ha medido a través de la varianza condicional de la tasa de inflación, utilizando la metodología de series de tiempo GARCH. Entre las principales investigaciones se encuentran las realizadas por Grier y Perry (1998) para los países del G-7; Benati y Surico (2008) para la economía estadounidense; Cogley (2009) para la economía americana; Fountas $(2001,2010)$ para el Reino Unido y el G-7; Fountas et al. (2004) para una muestra de cinco países europeos; Kontonikas (2004) para el Reino Unido; Conrad y Karanasos (2005) para Estados Unidos, el Reino Unido y Japón; Thornton (2007) para una muestra de doce economías emergentes; Grimme et al. (2012) para Estados Unidos, y Caporale et al. (2010) para la zona euro. En general, los resultados de estas investigaciones coinciden con la hipótesis de Friedman-Ball.

Por el contrario, autores como Pourgerami y Maskus (1978) y Ungar y Zilberfarb (1993) cuestionan el cumplimiento de la hipótesis de
Friedman-Ball; argumentan que en un contexto de crecimiento sostenido de los precios, los agentes económicos estarían más dispuestos en invertir recursos financieros para pronosticar la inflación; por lo tanto, se genera una relación inversamente proporcional entre la inflación y su incertidumbre. Estos autores no proporcionan evidencia empírica sobre su argumentación.

De igual forma, se ha investigado la posibilidad de una relación de retroalimentación, donde la incertidumbre inflacionaria también afecta la tasa de inflación. Este es el caso de la investigación realizada por Cukierman y Meltzer (1986), donde se formuló un modelo donde el Banco Central, para enfrentar un incremento en la incertidumbre inflacionaria, se predispone a intervenir de manera oportunista, con el propósito de aprovechar esta situación de volatilidad y así crear sorpresas inflacionarias a través de la implementación de políticas monetarias expansivas. Cuando existe un incremento en la inflación provocado por un aumento en la incertidumbre inflacionaria, los agentes económicos no son capaces de identificar la naturaleza de este incremento en los precios y, generalmente, intuyen que ello está relacionado con las preferencias del Banco Central o a un cambio en la oferta monetaria. De acuerdo con dichos autores, la incertidumbre inflacionaria es lo que motiva a la autoridad monetaria a generar sorpresas inflacionarias. En esta misma línea, Cukierman (1992) sostiene que la inflación y su incertidumbre exhiben una relación positiva, y que la dirección de causalidad va de la primera a la segunda (hipótesis de Cukierman-Meltzer); en consecuencia, para estos autores un efecto causal positivo de la incertidumbre de la inflación sobre la tasa de crecimiento de los precios es evidencia de un banco central oportunista.

En oposición al argumento anterior, Holland (1995) sostiene que cuando la incertidumbre inflacionaria aumenta como resultado de un crecimiento de la inflación, el Banco Central responderá contrayendo el crecimiento de la oferta monetaria para eliminar la incertidumbre inflacionaria y el efecto negativo asociado sobre el crecimiento económico; así, de acuerdo con la hipótesis de Holland, el efecto 
causal negativo de la incertidumbre inflacionaria sobre la inflación es evidencia de un Banco Central estabilizador.

Entre los principales trabajos empíricos revisados se encuentran los de Baillie et al. (1996), Grier y Perry (1998, 2000), Daal et al. (2004), Fountas (2010), Bojanic (2013), Caballero y Caballero (2015), Thornton (2008), Perrotini y Rodríguez (2012) y Conrad y Karanasos (2005). En general, la evidencia empírica aportada por estos autores es mixta y no concluyente en favor de alguna de las hipótesis mencionadas; ello muestra que el efecto causal entre la incertidumbre inflacionaria sobre la inflación promedio es específica de cada país, lo cual es un indicador de que cada banco central ajusta sus tasas de crecimiento monetario de forma diferente ante la incertidumbre nominal, dependiendo de sus preferencias relativas sobre la estabilización de la inflación.

Cuando Grier y Perry (1998) y Conrad y Karanasos (2005) analizaron las razones institucionales por las que el aumento de precios respondía a un incremento en la incertidumbre inflacionaria, los resultados variaron entre países. Aquellos asociados con bancos centrales cuya respuesta era oportunista se caracterizaban por una menor independencia de su banco central, en comparación con los países cuyo instituto central tenía una respuesta estabilizadora. Por su parte, los resultados de Fountas et al. (2006) en general son consistentes con la clasificación de la independencia de los bancos centrales realizada por Alesina y Summers (1993); así, en los países donde el banco central cuenta con menor independencia, se cumple la hipótesis de Cukierman-Meltzer y se evidencia que dicho comportamiento corresponde a la actuación de un banco central oportunista. Por el contrario, en el caso de los países donde el banco central cuenta con un mayor grado de independencia, la evidencia empírica confirma la hipótesis de un banco central estabilizador.

Otro índice comúnmente utilizado para identificar la independencia de un banco central es el provisto por Cukierman et al. (1992), que posteriormente fue actualizado por Polillo y Guillén (2005).
Utilizando este índice, Thornton (2008) encuentra que los bancos centrales que exhiben un comportamiento estabilizador, en el sentido de la hipótesis de Holland, son generalmente clasificados como autoridades centrales con mayor independencia.

Entre los trabajos empíricos sobre el nexo entre inflación e incertidumbre inflacionaria para el caso de la economía mexicana se encuentran los de Grier y Grier (2006) y de Thornton (2007); este último analiza doce economías emergentes entre las que se encuentra México; Fang et al. (2010) utilizan regresiones por cuantiles para datos de sección cruzada de 152 países, incluyendo a México; por su parte, Perrotini y Rodríguez (2012) examinan la relación entre inflación e incertidumbre inflacionaria mediante un modelo GARCH-M, con datos anuales para México.

En el estudio de Grier y Grier (2006) se estima un modelo GARCH-multivariado para la economía mexicana en el periodo comprendido entre enero de 1972 y diciembre de 2000. Los autores encuentran evidencia empírica que apoya la hipótesis de Friedman-Ball, por cuanto la inflación rezagada resulta ser positiva y altamente significativa, aunque no desarrollan la prueba sobre el efecto causal de incertidumbre inflacionaria en la inflación promedio; de igual forma se indaga la existencia de efectos no lineales de la tasa de inflación sobre la incertidumbre inflacionaria.

La investigación de Thornton (2007) se basa en una muestra de doce economías emergentes, incluyendo la economía mexicana, para el periodo comprendido entre enero de 1957 y diciembre de 2005, y se utiliza un modelo AR-GARCH. Los resultados para la economía mexicana muestran el cumplimiento de la hipótesis de Friedman-Ball, y al examinar la relación causal entre la incertidumbre nominal y la inflación se encontró que fue de tipo negativo, lo que evidencia el cumplimiento de la hipótesis de un banco central estabilizador planteada por Holland un incremento en: la incertidumbre inflacionaria conduce a una menor inflación promedio.

En el caso del trabajo de Fang et al. (2010), se utilizan regresiones por cuantiles para datos de sección cruzada de 152 países, incluyendo a 
México, para el periodo 1993-2003. Los autores emplean la media y la desviación estándar, la media y la variación relativa o la mediana y la desviación de la mediana de la tasa de inflación para medir la inflación y su variabilidad. Sus resultados apoyan tanto la hipótesis de Friedman-Ball como la hipótesis de Cukierman-Meltzer para el modelo de cuantiles paramétricos. En el primer caso, los resultados muestran que una mayor inflación está asociada con una mayor variabilidad en la inflación; en tanto que en el segundo caso (hipótesis de Cukierman y Meltzer) la variabilidad de la inflación incrementa la inflación.

Por último, en el estudio de Perrotini y Rodríguez (2012) se emplea un modelo GARCH en media, con datos anuales para la economía mexicana en el periodo 1929-2009. El modelo presenta evidencia a favor del cumplimiento de la hipótesis de Friedman-Ball, lo que significa que la volatilidad aumenta en proporción a la inflación rezagada un periodo, pero también se encuentra evidencia del incumplimiento de la hipótesis de Cukierman-Meltzer; en consecuencia, los datos muestran consistencia con la hipótesis de un banco central estabilizador, evidenciando de esta manera que el Banco de México intenta contrarrestar esta incertidumbre al contraer su oferta monetaria.

\section{METODOLOGÍA ECONOMÉTRICA}

Las investigaciones realizadas sobre la inflación de la economía mexicana no capturan su componente de variación estacional, debido a la naturaleza anual de los datos. Con el fin de subsanar esta falencia, en esta investigación se considera el efecto estacional en el valor medio de la inflación, utilizando para ello un modelo SARMA; a su vez, se calibran modelos GARCH, los cuales permiten identificar la incertidumbre inflacionaria, y posteriormente se emplean los modelos GJR-GARCH-M y E-GARCH-M para calcular la asimetría de los impactos positivos y negativos presentes en la varianza condicional. Estos modelos permiten, además, estimar la relación de retroalimentación entre la inflación y su incertidumbre. Finalmente, se incorpora el impacto que tienen los choques estructurales en la incertidumbre inflacionaria.

El enfoque Box-Jenkins para construir modelos lineales de series de tiempo se basa en dos grandes principios: el primero, el principio de parsimonia, consiste en elegir siempre el modelo más sencillo que sea suficientemente representativo respecto a los datos; el segundo, el principio del mejoramiento iterativo, consiste en partir de un modelo sencillo y factible, al cual se harán mejoramientos sucesivos hasta llegar a un modelo satisfactorio. El modelo de series de tiempo estándar de la inflación presenta la siguiente especificación:

$\pi_{t}=\gamma_{0}+\sum_{i=1}^{p} \gamma_{i} \pi_{t-i}+\sum_{i=0}^{q} \theta_{i} \varepsilon_{t-i}+\sum_{i=1}^{P} \phi_{i} \pi_{t-i s}+\sum_{i=0}^{Q} \delta_{i} \varepsilon_{t-i s}+\varepsilon_{t}$

Donde $\pi$ representa la tasa de inflación calculada como un cambio porcentual, $\pi_{t-1}$ son los valores rezagados de la tasa de inflación, $\Pi_{t-i s}$ son los valores autorregresivos estacionales de longitud, $s, \varepsilon_{\mathrm{t}}$ es el término de error que se asume como ruido blanco, mientras que $\varepsilon_{\mathrm{t}-\mathrm{i}}$ representa las medias moviles ordinarias y $\varepsilon_{\mathrm{t}-\mathrm{is}}$ representa las medias móviles estacionales. La ecuación [1] indica un modelo multiplicativo de la forma $\operatorname{ARIMA}(p, d, q)(P, D, Q)_{s}$. Estos modelos permiten capturar el componente estacional que se encuentra presente en serie de tiempo de la tasa de inflación; además, con ello se logra gran versatilidad y la posibilidad de generar ajustes que permiten una correcta interpretación del proceso estocástico. A continuación se ejemplifica la interpretación de un modelo $\mathrm{AR}(1)$ y $\mathrm{MA}(1)$, ambos con componente no estacional y estacional mensual.

Para garantizar que un modelo SARIMA se ajuste correctamente a los datos, se debe identificar el coeficiente de determinación y el promedio de la suma de residuos cuadráticos obtenidos mediante la metodología de mínimos cuadrados ordinarios generalizados. Atendiendo al principio de parsimonia, se sugiere utilizar el criterio de información de Akaike (AIC, por sus siglas inglés) o el criterio de información bayesiano (BIC, por sus siglas inglés); 
Figura 1.

Proceso autorregresivo integrado y de media móvil estacional (SARMA)

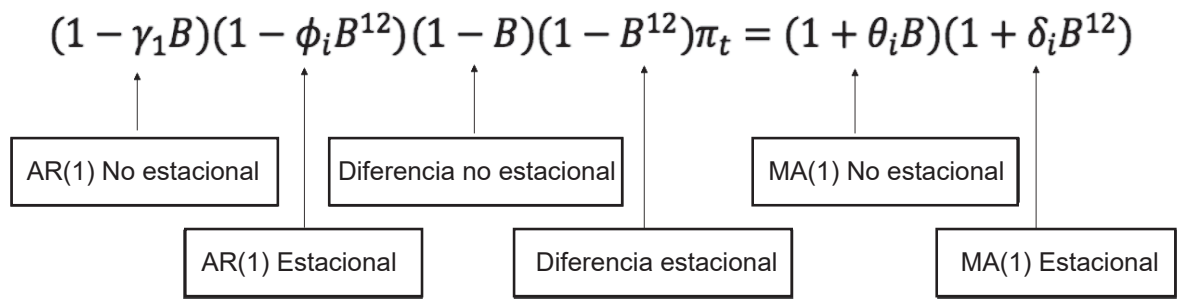

Fuente: elaboración de los autores.

posteriormente, se procede a identificar la volatilidad presente en la serie, lo cual permitirá construir la varianza condicional o, en otras palabras, la incertidumbre inflacionaria. Bollerslev (1986) introdujo el modelo GARCH que se especifica de la siguiente manera:

$h_{t}=\alpha_{0}+\sum_{i=1}^{q} \alpha_{i} \varepsilon_{t-i}^{2}+\sum_{i=1}^{p} \beta_{i} h_{t-i}$

Donde $\alpha_{0}$ representa la varianza condicional media, $\varepsilon_{\mathrm{t}-\mathrm{i}}^{2}$ son las noticias sobre la incertidumbre inflacionaria de los periodos pasados, $h_{t-i}$ son las varianzas condicionales de los periodos pasados y $\varepsilon_{\mathrm{t}}$ es el término de perturbación estocástica que se comporta como un ruido blanco. El proceso GARCH $(p, q)$ debe cumplir las siguientes condiciones: $\alpha_{0}>0, \alpha_{i} \geq 0, \beta_{i} \geq 0$, además de ser estacionario en sentido amplio, es decir, $\alpha_{i}+\beta_{i}<1$.

Una vez se cuenta con la media y varianza condicional $^{1}$, se emplea el método GARCH-M, el cual provee a la media de una secuencia que depende de su propia desviación estándar condicional $\left(\sqrt{h_{t}}=\frac{\varepsilon_{t}}{v_{t}}\right)$. Esta clase de modelos es particularmente adecuado

$1 \quad$ Los modelos pueden ser lineales en media y varianza (modelo clásico de regresión lineal múltiple, los modelos ARMA) o no lineales en media, pero lineales en varianza (modelos bicorrelacionados). Otros modelos también pueden clasificarse como lineales en media, pero no lineales en varianza (extensiones del modelo GARCH). Finalmente, los modelos pueden ser no lineales ni en media ni en varianza (los modelos de umbral híbrido con errores $\mathrm{GARCH}$ ) (Brooks, 2008, p. 380). para el estudio de causalidad y retroalimentación entre una variable y su volatilidad. Si la varianza condicional es constante $\left(\alpha_{1}=\alpha_{2}=\alpha_{3}=\ldots=\alpha_{q}=0\right)$, el modelo GARCH-M degenera en el caso más tradicional de una varianza condicional constante. Los modelos GARCH-M permiten probar las hipótesis de Friedman-Ball/Pourgerami y Maskus, y, simultáneamente, comprobar el cumplimiento de la hipótesis Cukierman-Meltzer/Holland. La idea consiste en utilizar la varianza condicional para explicar la tasa de inflación en la primera ecuación, y la tasa de inflación rezagada de un periodo para determinar la incertidumbre inflacionaria en la segunda ecuación (Kontonikas, 2004). Lo anterior permite estimar una relación de retroalimentación simultanea entre la inflación y la incertidumbre inflacionaria. El modelo se describe a través de las siguientes ecuaciones:

$$
\begin{aligned}
& \pi_{t}=\gamma_{0}+\sum_{i=1}^{p} \gamma_{i} \pi_{t-i}+\sum_{i=1}^{q} \theta_{i} \varepsilon_{t-i}+\sum_{i=1}^{P} \phi_{i} \pi_{t-i s}+ \\
& \sum_{i=1}^{Q} \delta_{i} \varepsilon_{t-i s}+\varphi \sqrt{h_{t}}+\varepsilon_{t}
\end{aligned}
$$

$$
h_{t}=\alpha_{0}+\sum_{i=1}^{q} \alpha_{i} \varepsilon_{t-i}^{2}+\sum_{i=1}^{p} \beta_{i} h_{t-i}+\delta \pi_{t-i}
$$

Las ecuaciones [3] y [4] son el modelo SARMA y el modelo GARCH-M, con la incorporación de la desviación estándar condicional y de la 
tasa de inflación rezagada en un periodo; el coeficiente $\varphi$ representa el efecto de la incertidumbre inflacionaria sobre la inflación promedio. Un valor positivo y estadísticamente significativo de este es interpretado como evidencia en favor de la hipótesis de Cukierman-Meltzer (1986), donde se establece que a una mayor incertidumbre sobre la postura de la política monetaria y, por tanto, de la tasa de inflación, la autoridad central tiene un incentivo para sorprender al público y aplicar una política monetaria excesivamente expansiva, anticipándose a las ganancias de producción; en este caso se dice que el banco central es oportunista. Entre tanto, un valor negativo indicaría que se cumple la hipótesis de estabilización de Holland (1993a, 1993b, 1995), la cual establece que, en respuesta a una mayor incertidumbre, y temiendo la posibilidad de efectos en las variables reales por tal incertidumbre, el Banco Central podría tratar de contrarrestar esta volatilidad mediante la contracción de su oferta monetaria y con ello reducir la inflación —-también denominado hipótesis de estabilización-. Por su parte, el estimador $\delta$ representa el coeficiente del rezago de la inflación y busca determinar el efecto nivel, es decir, el impacto que tiene la inflación sobre la incertidumbre inflacionaria - también denominada hipótesis de Friedman-Ball-

A partir de la evolución del modelo GARCH de Bollerslev (1986), se ha desarrollado un gran número de extensiones y variantes; algunas de ellas pueden capturar la no linealidad que exhiben las variables financieras y estimar el posible efecto de apalancamiento ${ }^{2}$, es decir, tratan los choques positivos y negativos de manera asimétrica. Para identificar cuándo es apropiado utilizar este tipo de modelos, Engle y Ng (1993, p. 1757) propusieron un conjunto de pruebas de asimetría para la incertidumbre de una serie de tiempo financiera, conocida como la prueba para sesgo de signo y tamaño (magnitud); en la práctica, la prueba es usualmente aplicada sobre los residuos de un

2 Para Brooks (2008, p. 405), un choque negativo en las series financieras puede causar un incremento en la incertidumbre, aun más que un choque positivo de la misma magnitud; a este efecto se le denomina apalancamiento. modelo ARMA-GARCH calculado sobre los cambios porcentuales de los datos; para ello se define $\mathrm{S}_{\mathrm{t}-1}^{-}$ como una variable binaria que asigna el valor de 1 si $\hat{\varepsilon}_{\mathrm{t}-1}<0$, y un valor de 0 en caso contrario $\left(\mathrm{S}_{\mathrm{t}-1}^{+}\right.$ $\left.=1-S^{-}{ }_{t-1}\right)$, para capturar las observaciones con innovaciones negativas y positivas. A partir de esta variable dummy, deriva una prueba conjunta para medir el sesgo de signo y tamaño, expresada en la siguiente ecuación de regresión:

$\hat{\varepsilon}^{2}=\phi_{0}+\phi_{1} S_{t-1}^{-}+\phi_{2} S^{-}{ }_{t-1}{ }^{*} \widehat{\varepsilon}_{\mathrm{t}-1}+\phi_{3} S^{+}{ }_{\mathrm{t}-1}{ }^{*} \hat{\varepsilon}_{\mathrm{t}-1}+\mathrm{U}_{\mathrm{t}}$

Donde $\mathrm{V}_{\mathrm{t}}$ es un termino de perturbación independiente e idénticamente distribuido. Si choques positivos y negativos para $\hat{\varepsilon}_{\mathrm{t}-1}$ impactan de forma diferente sobre la varianza condicional, entonces $\phi_{1}$ deberá ser estadísticamente significativo; por su parte, el tamaño o la magnitud de los choques afectarán la volatilidad siempre y cuando $\phi_{2} \circ \phi_{3}$ presenten significancia estadística, donde tanto el signo como la magnitud del choque serán importantes. Adicionalmente, se formula una prueba de significancia estadística conjunta calculando $N^{*} R^{2}$ de la regresión anterior, que asintóticamente seguirá una distribución $\mathrm{X}^{2}$ con tres grados de libertad bajo la hipótesis nula de la no existencia de efectos asimétricos.

Muchas de las extensiones del modelo GARCH han surgido como una consecuencia de los problemas percibidos con los modelos $\mathrm{GARCH}(\mathrm{q}, \mathrm{p})$ estándar. El primero, como se ha mencionado, es el problema de la asimetría, donde las condiciones de no negatividad pueden ser violadas por el modelo estimado, y la única forma de evitar esta situación sería agregar restricciones artificiales sobre los coeficientes del modelo para forzarlos a que sean no negativos. El segundo problema es que los modelos GARCH no pueden considerar el efecto de apalancamiento o su versión invertida, aunque sean capaces de considerar la acumulación de la volatilidad y la leptocurtosis presente en la serie de tiempo.

En la tabla 1 se presentan las formulaciones asimétricas más utilizadas en la literatura para solventar las problemáticas expuestas: a) el modelo 
GARCH exponencial (EGARCH) propuesto por Nelson (1991); b) el modelo GJR-GARCH, llamado así en honor de sus autores: Glosten, Jagannathan y Runkle (1993).

El modelo E-GARCH presenta tres características especiales: a) la ecuación para la varianza condicional es de la forma no lineal; a pesar de la magnitud del $\ln \left(h_{t}\right)$, sus valores no pueden ser negativos; b) estos modelos utilizan el nivel de los valores estandarizados de la variable $\varepsilon_{\mathrm{t}-1}$, es decir, divididos por $\left(h_{t}\right)^{1 / 2}$; en este sentido, Nelson (1991) argumenta que esta estandarización permite una interpretación más natural del tamaño y la persistencia de los choques; c) permiten capturar el efecto apalancamiento: si $\varepsilon_{t-1} /\left(h_{t}\right)^{1 / 2}$ es positivo, el efecto del choque sobre el logaritmo de la varianza condicional es $\alpha_{1}+\gamma_{1}$; por el contrario, si es negativo, el efecto del choque será $-\alpha_{1}+\gamma_{1}$.

En el modelo GJR-GARCH y T-GARCH desarrollado independientemente por Zakoïan (1994) y Glosten et al. (1993), respectivamente, las buenas noticias $\varepsilon_{\mathrm{t}-1}>0 \mathrm{y}$ las malas noticias $\varepsilon_{\mathrm{t}-1}<0$ tienen diferentes efectos sobre la varianza condicional. Así, las buenas noticias tienen un impacto sobre $\alpha_{i}$, mientras que las malas noticias lo tienen sobre $\alpha_{i}+\gamma_{i}$. Si $\gamma_{i}>0$, las malas noticias incrementarán la incertidumbre; se dice entonces que existe un efecto apalancamiento para el i-esimo orden; a su vez, si $\gamma_{i} \neq 0$, el impacto de las noticias será asimétrico. La principal diferencia entre estos dos modelos es que el primero busca capturar el comportamiento de la varianza condicional, mientras que el segundo lo hace respecto a la desviación estándar condicional.

Para estimar los modelos descritos se utiliza la metodología de máxima verosimilitud, que busca los valores con mayor probabilidad, dados los datos reales; para ello se emplea una técnica iterativa que maximiza el logaritmo de la función de verosimilitud, lo cual significa que, dado un conjunto inicial de conjeturas para los parámetros estimados, los valores de los parámetros son actualizados en cada iteración hasta que se determina que un punto óptimo ha sido obtenido (Brooks, 2008, p. 398).

Dado que el término $\varepsilon_{\mathrm{t}}$ sigue una distribución gaussiana condicional sobre el pasado, la predicción de descomposición sobre el error de la función del logaritmo de verosimilitud del modelo condicional GARCH en los valores iniciales es:

$$
\begin{gathered}
\log L=\sum_{\mathrm{t}=1}^{\mathrm{T}} \mathrm{I}_{\mathrm{t}}=-\frac{\mathrm{T}}{2} \log (2 \pi)-\frac{1}{2} \sum_{\mathrm{t}=1}^{\mathrm{T}} \sigma^{2}{ }_{\mathrm{t}}-\frac{1}{2} \sum_{\mathrm{t}=1}^{\mathrm{T}} \frac{\varepsilon^{2}{ }_{\mathrm{t}}}{\sigma^{2}{ }_{\mathrm{t}}} \\
\text { Donde } \mathrm{I}_{\mathrm{t}}=-1 / 2\left(\log (2 \pi)+\log \sigma^{2}{ }_{\mathrm{t}}\right)-\left(\frac{1}{2}\right)\left(\frac{\varepsilon^{2}{ }_{\mathrm{t}}}{\sigma^{2}{ }_{\mathrm{t}}}\right)
\end{gathered}
$$

Tabla 1.

Extensiones de los modelos GARCH

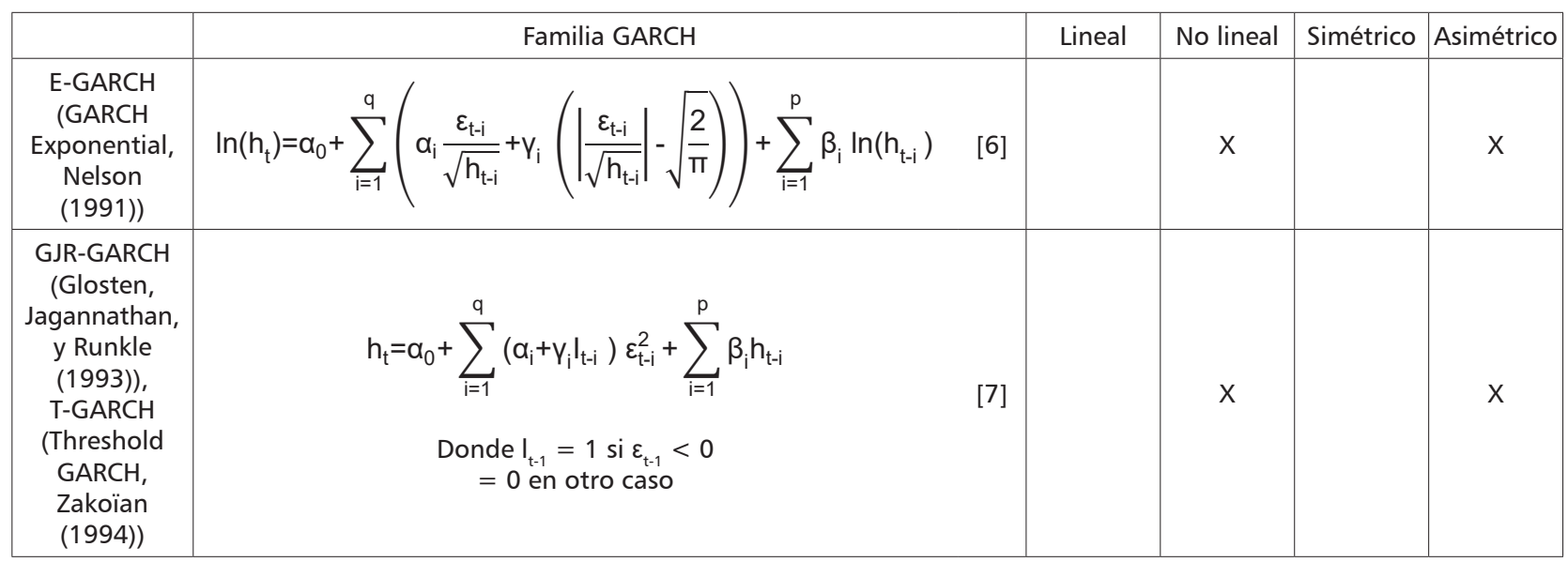

Fuente: elaboración de los autores. 
utilizado en la práctica: mediante un procedimiento computacional numérico se aproxima el valor exacto del logaritmo de verosimilitud ${ }^{3}$. Una vez que el logaritmo de verosimilitud es inicializado, este puede ser maximizado utilizando técnicas de optimización numérica; los más utilizados son el desarrollado por Berndt et al. (1974), conocido como el algoritmo $\mathrm{BHHH}$, y el algoritmo Marquardt; ambos son variaciones del método de Gauss-Newton (Press et al., 1992). Este algoritmo aproxima la matriz hessiana (H) utilizando únicamente la información de la primera derivada:

$-H(\theta) \approx B(\theta)=\sum_{\mathrm{t}=1}^{T} \frac{\partial \mathrm{I}_{\mathrm{t}}}{\partial \theta} * \frac{\partial \mathrm{I}_{\mathrm{t}}}{\partial \theta^{\prime}}$

Aun cuando se ha garantizado la correcta calibración de los estimadores del modelo GARCH, existen algunos aspectos del modelo que pueden ser mejorados con la finalidad de que este capture las características y la dinámica de la tasa de cambio del INPC. Estos aspectos se refieren a que, dado el largo periodo estudiado en esta investigación, podría estar presente el efecto que generan los quiebres estructurales.

\section{ESTIMACIÓN Y RESULTADOS EMPÍRICOS}

La parte empírica de este trabajo tiene como objetivo analizar el comportamiento de la inflación y la incertidumbre inflacionaria en México, para un periodo más amplio que los estudios previos, enero de 1969 a febrero 2017, utilizando ecuaciones en diferencias estocásticas cuyos estimadores serán calculados mediante la metodología de modelos híbridos SARMA-GARCH, que requieren un control metodológico y estadístico. Los datos empleados para desarrollar la estimación del modelo empírico corresponden al índice nacional de precios al consumidor (INPC) para el periodo mencionado, lo que representa 578 observaciones. Los datos

3 Para los valores iniciales de $\sigma_{\mathrm{t}}^{2}$, una elección práctica es: $\sigma_{t}^{2}=\varepsilon_{t}^{2}=1 / t \sum_{s=1}^{t} \varepsilon_{s=1}^{2}, t \leq 0$, donde los valores iniciales de $\varepsilon_{\mathrm{s}}$ son calculados como las perturbaciones de la regresión de $T_{t}$ sobre una constante. mensuales se obtuvieron del Banco de México, y el periodo de muestra se eligió considerando la disponibilidad de los datos.

\section{Análisis de la serie temporal}

Con base en la metodología Box-Jenkins, el análisis de la serie temporal comienza con la verificación preliminar de la estacionariedad del INPC y de su transformación algebraica (diferencias logarítmicas multiplicadas por cien para obtener los cambios porcentuales), también conocida como el cambio

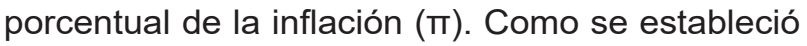
anteriormente, las variables económicas y financieras exhiben ciertas regularidades empíricas; en el caso de INPC, presenta una clara tendencia positiva, así como marcados quiebres estructurales, y al transformarla presenta una marcada volatilidad que no es constante a lo largo del tiempo (figura 2).

$$
\begin{aligned}
& \Pi=\log \left(\frac{I^{N P C} C_{t}}{\operatorname{INPC}_{t-1}}\right)=\log \left(1+\frac{I^{N P C} C_{t}-I N P C_{t-1}}{I N P C_{t-1}}\right)= \\
& \log \left(I N P C_{t}\right)-\log \left(I N P C_{t-1}\right)
\end{aligned}
$$

En el análisis gráfico se aprecia que el comportamiento mensual del INPC no parece estacionario, mientras que el cambio porcentual de la inflación sí parece serlo. Con la finalidad de determinar si la serie de tiempo del INPC y su cambio porcentual poseen una raíz unitaria, se realiza un análisis formal, es decir, se lleva a cabo la prueba DickeyFuller aumentada y Phillips-Perron para determinar la existencia de una raíz unitaria.

Con base en las pruebas de raíz unitaria de la tabla 2, se puede establecer que el INPC presenta un orden de integración 1 en sus tres versiones, mientras que la serie de tiempo del cambio porcentual de la inflación ( $\pi$ ) muestra un orden de integración de 0 , con intercepto, con tendencia e intercepto y sin las anteriores; adicionalmente, se generan las funciones de autocorrelación simple (FAC) y parcial (FACP) para determinar el proceso generador de datos del cual proviene la serie de tiempo. 
Gráfica 1.

INPC en niveles y en diferencias logarítmicas (inflación)

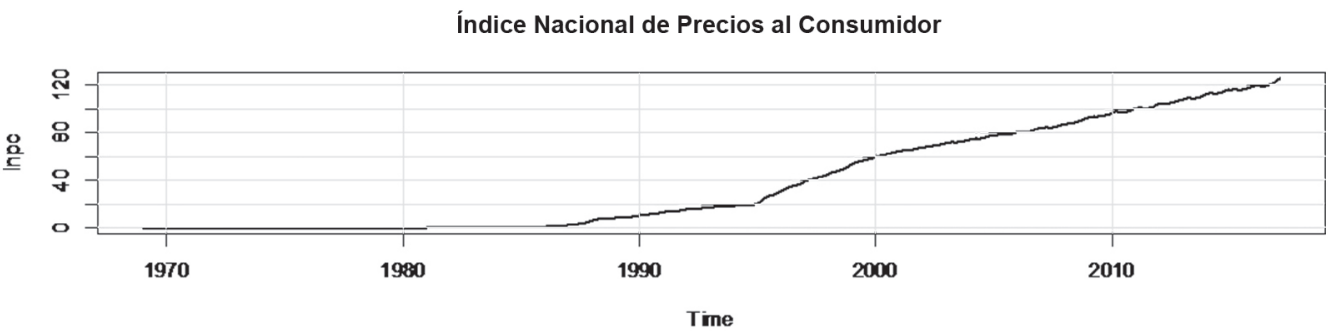

Cambio Porcentual de la Inflación (II)

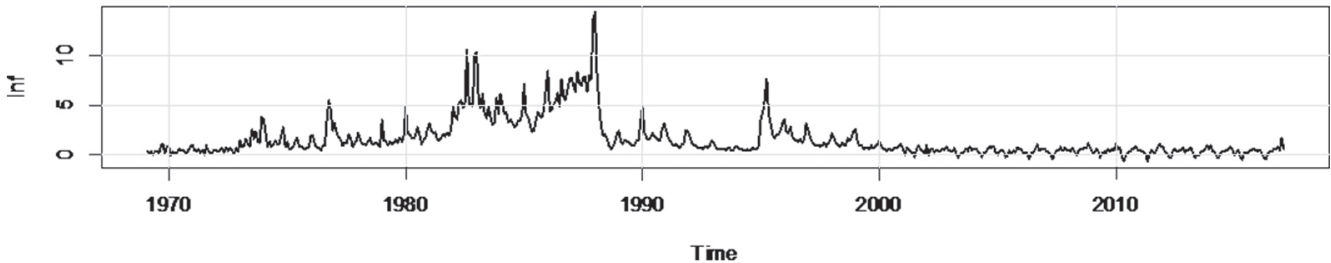

Fuente: elaboración de los autores con base en datos del IFS del FMI.

Tabla 2.

Pruebas de raíz unitaria

\begin{tabular}{|c|c|c|c|c|c|}
\hline \multirow[b]{3}{*}{ Intercepto } & \multicolumn{5}{|c|}{ INPC } \\
\hline & \multicolumn{2}{|c|}{ DFA } & \multicolumn{2}{|c|}{$\mathrm{PP}$} & Orden de integración \\
\hline & 4,1294 & 1,0000 & 4,5617 & 1,0000 & $\mathrm{I}(1)$ \\
\hline Tendencia e intercepto & $-1,8998$ & 0,6533 & $-2,0587$ & 0,5672 & $I(1)$ \\
\hline \multirow[t]{3}{*}{ Sin tendencia ni intercepto } & 6,3314 & 1,0000 & 7,8779 & 1,0000 & $I(1)$ \\
\hline & \multicolumn{5}{|c|}{$\Delta \log ($ INPC) $(\pi)$} \\
\hline & \multicolumn{2}{|c|}{ DFA } & \multicolumn{2}{|c|}{ PP } & Orden de integración \\
\hline Intercepto & $-4,3909$ & 0,0003 & $-5,4001$ & 0,0000 & $\mathrm{I}(0)$ \\
\hline Tendencia e intercepto & $-4,7631$ & 0,0006 & $-5,8187$ & 0,0000 & $I(0)$ \\
\hline Sin tendencia ni intercepto & $-3,3385$ & 0,0009 & $-4,0757$ & 0,0000 & $I(0)$ \\
\hline
\end{tabular}

Nota: se utilizó el criterio de información de Schwarz para la selección automática de los rezagos.

Fuente: elaboración de los autores.

El primer paso para modelar la variación de la volatilidad o la incertidumbre en una serie de tiempo es especificar una ecuación adecuada para la media condicional de $(\pi)$. Dada la ausencia de un modelo estructural de aceptación común para el cambio porcentual de la inflación, las especificaciones autorregresivas y de medias móviles tanto ordinarias como estacionales se han vuelto populares en la literatura empírica. En la figura 3 se puede identificar el patrón que representa el proceso estocástico y, de esta manera, proponer el modelo ARMA adecuado. De acuerdo con la literatura especializada, un patrón decreciente en ambas funciones evidencia un modelo con componentes tanto autorregresivos como de medias móviles; también se aprecia una marcada variación estacional, la cual está presente en el duodécimo mes de los primeros tres años. 

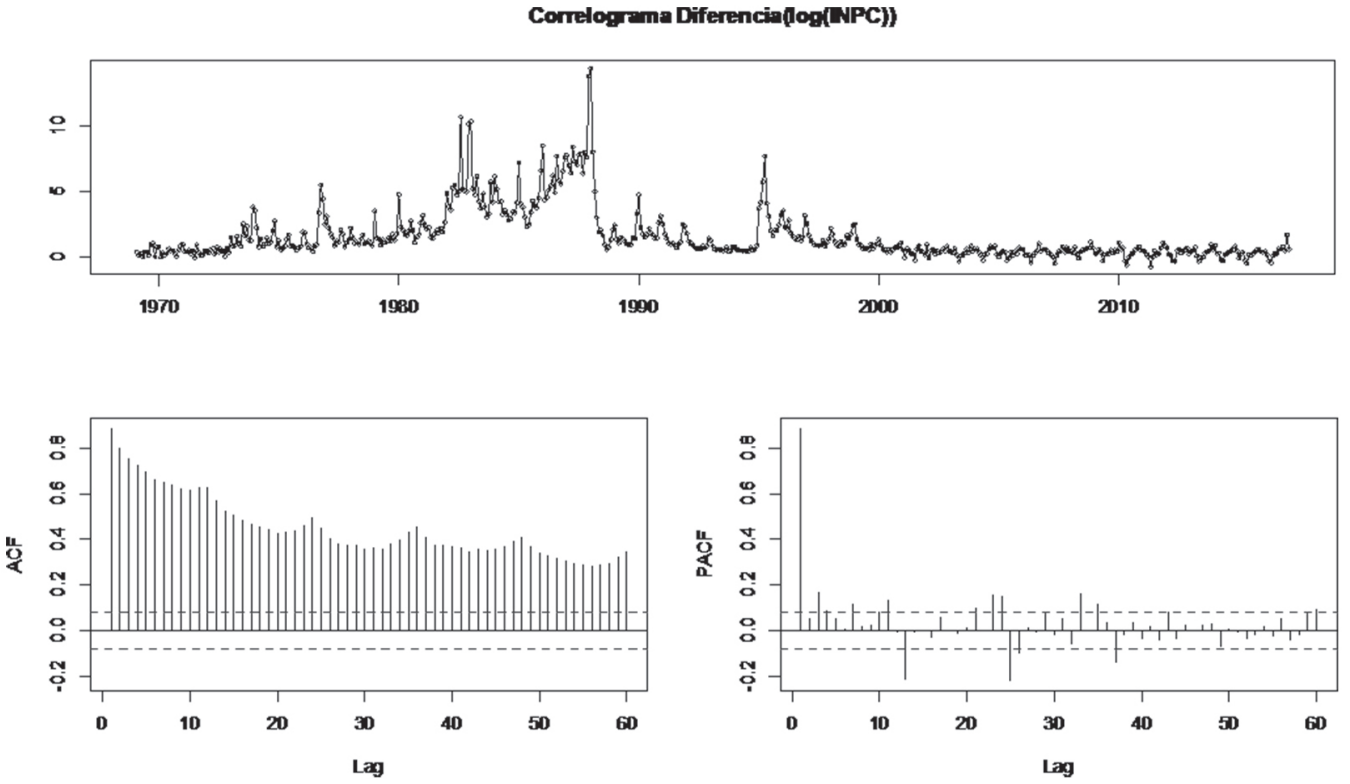

Fuente: elaboración de los autores con base en datos del IFS del FMI.

Actualmente existen algunos softwares ${ }^{4}$ que incluyen un algoritmo capaz de identificar el modelo SARMA óptimo con base en los criterios AIC o BIC; se debe señalar que este último siempre seleccionará un modelo más parsimonioso que el primero (Enders, 2015, p. 69). Para la serie del cambio porcentual de la inflación de México, se identifica que el modelo posee una estructura $\operatorname{SARMA}(2,0,1) \times(3,0,0)_{12}$, lo cual indica que la serie de tiempo de la inflación está conformada por dos componentes autorregresivos y uno de media móvil, tres de tipo ordinario, además de otros tres componentes autorregresivos de tipo estacional. En la tabla 1 se presentan los resultados de los coeficientes para las diferentes propuestas de procesos estocásticos. El primer modelo se ubica

4 Rob Hyndman (2008) ha desarrollado la librería forercast, que cuenta con la función auto.arima para definir los parámetros autorregresivos integrados y de medias móviles óptimos. como el óptimo, mientras que los seis modelos restantes agregan y eliminan un autorregresivo y una media móvil ordinaria, y en el caso de los rezagos estacionales se agrega y elimina un componente autorregresivo estacional.

De acuerdo con la metodología Box-Jenkins, en la siguiente etapa se debe realizar un análisis para diagnosticar la bondad de ajuste del modelo óptimo. En la tabla 3 se registran los principales estadísticos utilizados en la práctica, basados en la suma de residuos cuadráticos (SRC). Es evidente que el primer y séptimo modelo presentan los valores más bajos de este estadístico (RMSE), y que la mayoría de los coeficientes estimados son, en ambos casos, estadísticamente significativos; sin embargo, los criterios de información de Akaike y Bayesiano -este criterio proporciona el modelo más parsimonioso- indican que la opción óptima debe ser el primer y el quinto modelo. Se puede identificar que todos los coeficientes son 
Tabla 3.

Resultados de las regresiones SARMA (máxima verosimilitud)

\begin{tabular}{|c|c|c|c|c|c|c|c|}
\hline & \multicolumn{7}{|c|}{ Variable dependiente: $\Delta \log ($ INPC) } \\
\hline & Modelo 1 & Modelo 2 & Modelo 3 & Modelo 4 & Modelo 5 & Modelo 6 & Modelo 7 \\
\hline & $\begin{array}{c}\text { SARIMA } \\
(2,0,1) \times(3,0,0) \\
{[12]}\end{array}$ & $\begin{array}{c}\text { SARIMA } \\
(3,0,1) \times(3,0,0) \\
{[12]}\end{array}$ & $\begin{array}{c}\text { SARIMA } \\
(1,0,1) \times(3,0,0) \\
{[12]}\end{array}$ & $\begin{array}{c}\text { SARIMA } \\
(2,0,0) \times(3,0,0) \\
{[12]}\end{array}$ & $\begin{array}{c}\text { SARIMA } \\
(2,0,2) \times(3,0,0) \\
{[12]}\end{array}$ & $\begin{array}{c}\text { SARIMA } \\
(2,0,1) \times(2,0,0) \\
{[12]}\end{array}$ & $\begin{array}{c}\text { SARIMA } \\
(2,0,1) \times(4,0,0) \\
{[12]}\end{array}$ \\
\hline \multirow[t]{2}{*}{ C } & -- & -- & -- & -- & $1,6430^{* *}$ & -- & -- \\
\hline & -- & -- & -- & -- & 0,7446 & -- & -- \\
\hline \multirow[t]{2}{*}{$\begin{array}{l}\text { AR } \\
\text { (1) }\end{array}$} & $1,4113^{* * *}$ & $1,2359 * * *$ & $0,9065^{* * *}$ & $0,7965^{* * *}$ & $-0,0647^{* *}$ & 1,4331 ** & $1,4582^{* * *}$ \\
\hline & 0,181 & 0,2343 & 0,0213 & 0,0436 & 0,0275 & 0,1726 & 0,1605 \\
\hline \multirow[t]{2}{*}{$\begin{array}{l}\text { AR } \\
(2)\end{array}$} & $-0,441 * * *$ & $-0,3763^{* * *}$ & -- & $0,0877^{* * *}$ & $0,8546^{* * *}$ & $-0,4595^{* * *}$ & $-0,4838^{* * *}$ \\
\hline & 0,1642 & 0,1821 & -- & 0,0432 & 0,0254 & 0,1577 & 0,1461 \\
\hline \multirow[t]{2}{*}{$\begin{array}{l}\text { AR } \\
\text { (3) }\end{array}$} & -- & $-0,0933$ & -- & -- & -- & -- & -- \\
\hline & -- & 0,0571 & -- & -- & -- & -- & -- \\
\hline \multirow[t]{2}{*}{$\begin{array}{c}\text { MA } \\
\text { (1) }\end{array}$} & $-0,6562 * * *$ & $-0,4578 * * *$ & $-0,1430 * * *$ & -- & $0,8381 * * *$ & $-0,6719 * * *$ & $-0,7013^{* *}$ \\
\hline & 0,1615 & 0,2333 & 0,0489 & -- & 0,0521 & 0,1529 & 0,1407 \\
\hline \multirow[t]{2}{*}{$\begin{array}{c}\text { MA } \\
\text { (2) }\end{array}$} & -- & -- & -- & -- & $-0,1 \mathrm{t}$ & -- & -- \\
\hline & -- & -- & -- & -- & 0,0515 & -- & -- \\
\hline \multirow[t]{2}{*}{$\begin{array}{l}\text { SAR } \\
(12) \\
\end{array}$} & $0,1325^{* * *}$ & $0,1325^{* * *}$ & $0,1436^{* * *}$ & $0,1467^{* * *}$ & $0,1521 * * *$ & $0,1952^{* * *}$ & $0,1040^{* * *}$ \\
\hline & 0,0439 & 0,0438 & 0,0433 & 0,0433 & 0,0445 & 0,0428 & 0,0451 \\
\hline \multirow[t]{2}{*}{$\begin{array}{l}\text { SAR } \\
\text { (24) }\end{array}$} & $0,2503^{* * *}$ & $0,2495^{* * *}$ & $0,2614^{* * *}$ & $0,2609 * * *$ & $0,2524^{* * *}$ & $0,2885^{* * *}$ & $0,2167^{* * *}$ \\
\hline & 0,0416 & 0,0415 & 0,0411 & 0,0412 & 0,0417 & 0,0412 & 0,0432 \\
\hline \multirow[t]{2}{*}{$\begin{array}{l}\text { SAR } \\
(36)\end{array}$} & $0,2194^{* * *}$ & $0,2173^{* * *}$ & $0,2207 * * *$ & $0,2206^{* * *}$ & $0,2085^{* * *}$ & -- & $0,2061 * * *$ \\
\hline & 0,0423 & 0,0424 & 0,0422 & 0,0422 & 0,0429 & -- & 0,0429 \\
\hline \multirow[t]{2}{*}{$\begin{array}{l}\text { SAR } \\
(48)\end{array}$} & -- & -- & -- & -- & -- & -- & $0,1204^{* * *}$ \\
\hline & -- & -- & -- & -- & -- & -- & 0,0435 \\
\hline AIC & 2,4930 & 2,4937 & 2,5012 & 2,5061 & 2,4906 & 2,5207 & 2,4985 \\
\hline $\mathrm{BIC}$ & 2,5408 & 2,5495 & 2,5409 & 2,5459 & 2,5543 & 2,5598 & 2,5552 \\
\hline $\log V_{1}$ & $-665,88$ & $-663,8184$ & $-670,3384$ & $-670,4124$ & $-663,2329$ & $-689,4683$ & $-651,3793$ \\
\hline RMSE & 0,8044 & 0,8024 & 0,8068 & 0,8112 & 0,8005 & 0,8005 & 0,7947 \\
\hline
\end{tabular}

Nota: los errores estándar se encuentran en paréntesis; ${ }^{*} 0,10 ; * * 0,05 ; * * * 0,01$.

Fuente: elaboración de los autores.

estadísticamente significativos a un nivel de confianza de, al menos, $5 \%$.

Debido a que el modelo es explicado por procesos estocásticos estacionarios, se garantiza que es estable. Lo expuesto anteriormente corrobora los resultados exhibidos por el algoritmo de detección del modelo óptimo, razón por la cual será utilizado para modelar sus perturbaciones estocásticas e identificar la existencia de varianzas condicionales mediante un modelo GARCH. 
A pesar de que los modelos SARMA son flexibles en el desarrollo de pronósticos, no son capaces de manejar la volatilidad y la no linealidad que suele estar presente en las series de tiempo económicas. Así, para identificar la posible volatilidad existente en la serie de tiempo del cambio porcentual de la inflación, se efectúa una regresión de la diferencia logarítmica de la variable sobre una constante y se obtienen sus perturbaciones. De los datos se obtuvo la siguiente regresión por MCO:

$\Pi_{t}=\beta_{1}+u_{t}$

Donde $\pi_{t}$ es el cambio porcentual de la inflación y $u_{t}$ es el termino de error aleatorio:

$$
\begin{gathered}
\hat{\Pi}_{t}=1,5601 \\
t=18,9255
\end{gathered}
$$

A partir de la regresión anterior se obtuvieron los términos de perturbación $\left(\varepsilon_{\mathrm{t}}\right)$ y se elevaron al cuadrado $\left(\varepsilon_{t}^{2}\right)$ para utilizarlos como una medida de volatilidad. Al ser un valor cuadrático, este será alto en periodos en los que se experimentan grandes cambios en los precios y pequeño cuando suceden cambios moderados (gráfica 3).

Para comprobar formalmente que existe un efecto GARCH en el cambio porcentual de la inflación, se utiliza la prueba propuesta por McLeod y Li (1983). Empleando una muestra de residuos $T$, bajo la hipótesis nula de ausencia de errores GARCH, el estadístico de prueba $T^{\star} R^{2}$ converge en una distribución $X^{2}$ con $q$ grados de libertad. Si $T^{*} R^{2}$ es suficientemente grande frente a los niveles de significancia determinados, el rechazo de la hipótesis nula indicaría que existe evidencia estadística de efectos GARCH; por otra parte, si $T^{*} R^{2}$ es suficientemente bajo, es posible concluir que no hay efectos GARCH.

A pesar de que la estimación de un modelo GARCH permite modelar la volatilidad del cambio porcentual de la inflación, es necesario tomar en cuenta el papel que tienen los cambios estructurales, ya que pueden alterar la varianza e incrementar la incertidumbre inflacionaria. Utilizando una prueba de cambio estructural, se identifican tres puntos de quiebre: el primero se ubica en el

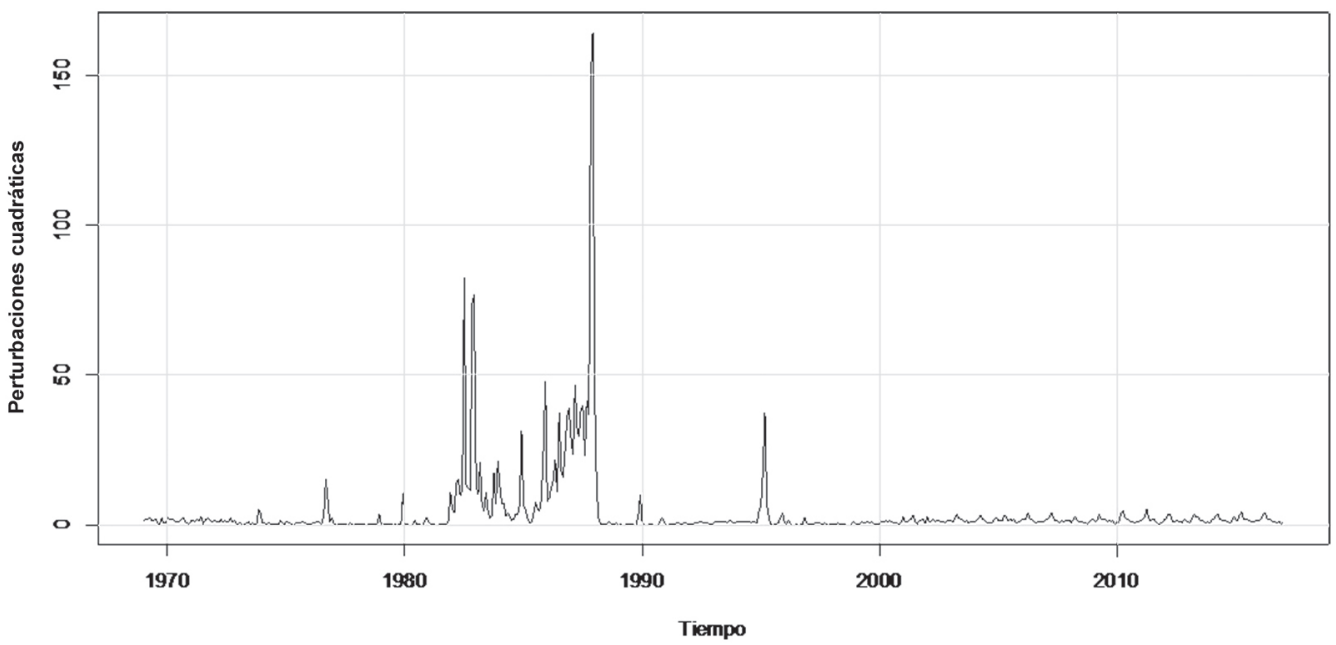

Fuente: elaboración de los autores con base en datos del IFS del FMI. 


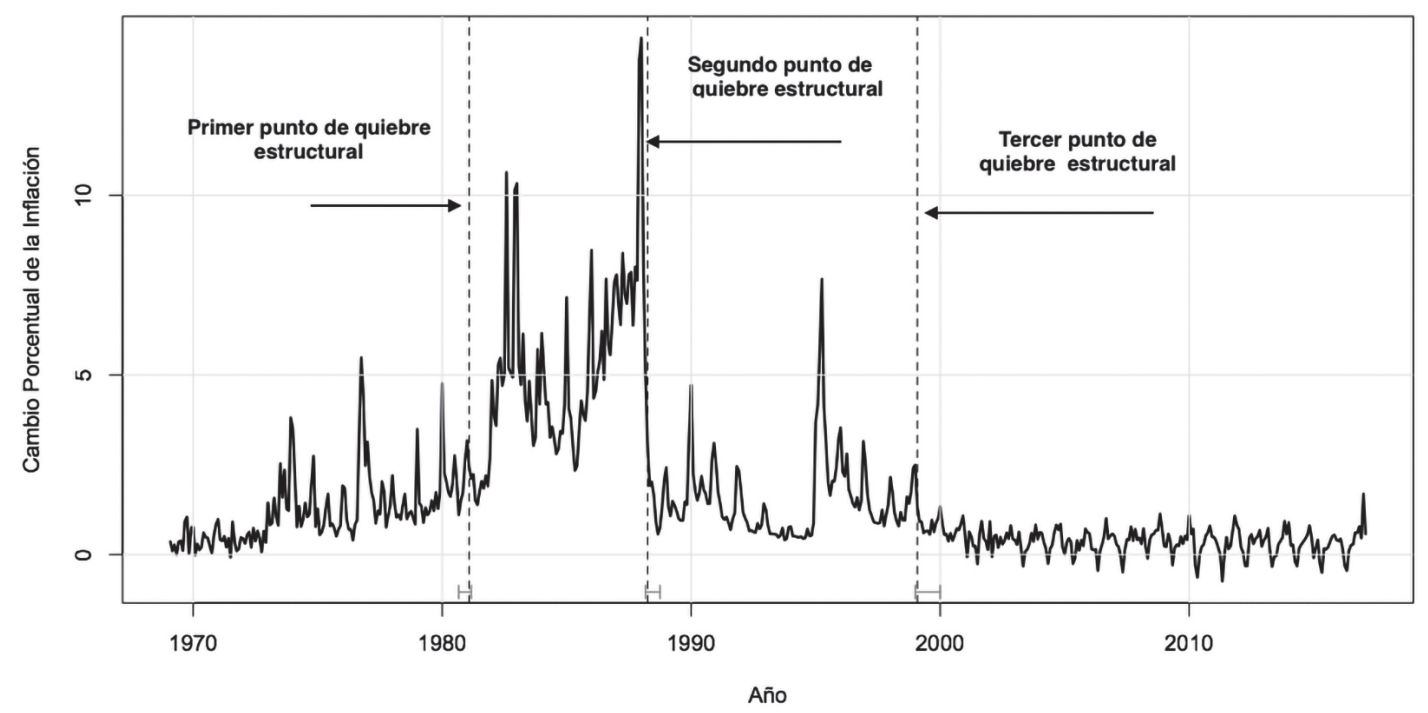

Fuente: elaboración de los autores con base en datos del IFS del FMI.

segundo mes de 1981, el segundo en el cuarto mes de 1988 y el tercero en el segundo mes de $1999^{5}$ (gráfica 4). Estos resultados coinciden con uno de los dos quiebres estructurales analizados por Broto (2008, p. 23), en cuyo estudio sobre metas de inflación para América Latina encuentra quiebres estructurales en 1983 y 1988. También hay coincidencias muy precisas en dos de las tres fechas de quiebres estructurales encontradas por Capistran y Ramos-Francia (2006, p. 20), que utilizaron el BIC en su investigación sobre la dinámica de la inflación en América Latina; dichas fechas son: diciembre de 1983, marzo de 1988 y febrero de 1999.

Con estos resultados, se construye una variable dummy para corregir el efecto de los choques estructurales sobre el cambio porcentual de la inflación; se evidencia que esta variable, que captura los cambios estructurales, es estadísticamente significativa, con un nivel de confianza del $1 \%$. Con la introducción de una variable dicótoma se recogieron los cambios de la varianza detectados mediante la asignación del valor 0 antes y después del cambio estructural, y 1 cuando se produce exactamente un punto de quiebre en la varianza de la serie:

$\varepsilon_{\mathrm{t}}^{2}=0,9551+0,7342 \varepsilon^{2}{ }_{\mathrm{t}-1}-0,2238 \varepsilon^{2}{ }_{\mathrm{t}-2}$ $+0,1337 \varepsilon_{\mathrm{t}-3}^{2}+0,1311 \varepsilon_{\mathrm{t}-4}^{2}-14,3134$ dummy

$$
(2,39)(17,90)(-4,31)(2,52)(3,15)(-2,60)
$$

Una vez comprobada la existencia de un efecto GARCH, y consideradas las fechas precisas que condujeron a los diferentes quiebres estructurales en la varianza, para explicar la volatilidad del cambio porcentual de la inflación, la estrategia empírica consiste en la estimación de diferentes modelos para medir su ajuste.

$5 \quad$ El software estadístico R-Project 3.4 ha desarrollado la librería strucchange, cuya función breakpoints permite definir los puntos de quiebre estructural (Zeileis et al., 2002). 


\section{Modelo GARCH y GARCH-M}

A continuación se presenta la estimación de los modelos SARMA-GARCH y SARMA-GARCH-M, los cuales representan regresiones para la tasa de inflación promedio (tabla 4), y están adicionados por la desviación estándar condicional; por otro lado, se presenta un modelo para la incertidumbre inflacionaria (varianza condicional), que se encuentra incrementada por la inflación rezagada un periodo y la variable dummy de quiebres estructurales. Este sistema de ecuaciones permite probar simultáneamente las hipótesis planteadas; así, por un lado, la ecuación de la media para probar la hipótesis de Cukierman-Meltzer o Holland y, por otro, la ecuación de la varianza que permite aceptar o rechazar la hipótesis de Friedman-Ball.

Las estimaciones presentadas en tabla 4 se desarrollaron mediante el método de cuasi máxima verosimilitud ( $Q M L)$, y la condición de estacionariedad $\alpha_{1}+\beta_{1}<1$ se verifica en ambos modelos. La mayoría de los estimadores son estadísticamente significativos al $1 \%$, tanto para la media como para la varianza condicional del primer y segundo modelo. También se aprecia que en el segundo modelo la inclusión de la variable DUM (dummy) es negativa y estadísticamente significativa al $5 \%$, lo que permite establecer la existencia de un efecto negativo de los choques estructurales de 1981, 1988 y 1999.

En el segundo modelo se comprueba que la inflación rezagada en un periodo impacta de manera positiva la incertidumbre inflacionaria a un nivel de $0,080(8 \%)$ significativo al $1 \%$; ello representa evidencia estadística en favor de la hipótesis de Friedman-Ball, que afirma que un incremento en la tasa de inflación causa una mayor volatilidad inflacionaria. Los resultados de dicho modelo también comprueban que el impacto del error estándar condicional (incertidumbre) sobre la inflación promedio es de $-0,078(-7,8 \%)$, que es negativo y significativo al $1 \%$, lo que significa que niveles de incertidumbre inflacionaria más altos conducirán a niveles de inflación promedio menores. Esto último se constituye como evidencia a favor de un banco central estabilizador (Holland, 1995) puesta a una mayor incertidumbre inflacionaria, y temiendo los posibles efectos reales de tal incertidumbre, contrarresta esta volatilidad mediante la contracción de la oferta monetaria; por tanto, no se encuentra evidencia para el caso de la economía mexicana de un banco central oportunista para el periodo analizado.

Con base en el trabajo desarrollado por Dincer y Eichengreen (2010, p. 218), en el cual se mide la transparencia e independencia de 100 bancos centrales, se observa que estos se han movido de forma constante en la dirección de una mayor transparencia e independencia en el tiempo; asimismo, muestran que la incertidumbre de la inflación se ve afectada significativamente por la transparencia y la independencia del banco central. Respecto a la independencia de un banco central, los autores utilizan las definiciones seguidas por Cukierman et al. (1992), que basan sus medidas de independencia central en una escala de cero a uno (menor independencia y mayor independencia respectivamente). La escala refleja la independencia del gobernador del banco central, su independencia en la formulación de políticas, su objetivo o mandato y la rigurosidad de los límites de sus préstamos al sector público. En su tabla comparativa de independencia ponderada de los bancos centrales para la región de América Latina y el Caribe, respecto a las economías más grandes, los resultados de Dincer y Eichengreen (2010) ponen a Chile y México, con una clasificación de 0,69 y 0,63, respectivamente, como las economías cuyos bancos centrales son más independientes. Esto es consistente con la hipótesis de un banco central estabilizador y demuestra que la mayor independencia del Banco de México le permite reducir el crecimiento de la oferta monetaria cuando la incertidumbre inflacionaria aumenta como resultado de un crecimiento de la inflación, para eliminar la incertidumbre inflacionaria $y$ el efecto negativo asociado sobre el crecimiento económico.

$6 \quad$ Los resultados obtenidos son consistentes con los desarrollados por Thornton (2007) y Perrotini y Rodríguez (2012) para el caso mexicano. 
Tabla 4.

Resultados de las Regresiones GARCH y GARCH-M (QML)

\begin{tabular}{|c|c|c|}
\hline \multicolumn{3}{|c|}{$\Delta \log (\mathrm{INPC})=(\pi)$} \\
\hline & Modelo 1 & Modelo $2^{\mathrm{a}}$ \\
\hline Var. Indep. & GARCH $(1,1)$ & $\mathrm{GARCH}(1,1)-\mathrm{M}$ \\
\hline \multicolumn{3}{|c|}{ ECUACIÓN PARA LA MEDIA CONDICIONAL } \\
\hline \multirow{2}{*}{$\operatorname{AR}(1)$} & 1,4072 & 1,3585 \\
\hline & $(0,1228)^{* * *}$ & $(0,1075)^{* * *}$ \\
\hline \multirow{2}{*}{$A R(2)$} & $-0,4504$ & $-0,3904$ \\
\hline & $(0,1050)^{* * *}$ & $(0,0958)^{* * *}$ \\
\hline \multirow{2}{*}{$\mathrm{MA}(1)$} & $-0,7811$ & $-0,7290$ \\
\hline & $(0,0863)^{* * *}$ & $(0,0817)^{* * *}$ \\
\hline \multirow{2}{*}{ SAR (12) } & 0,2450 & 0,2286 \\
\hline & $(0,0464)^{* * *}$ & $(0,0415)^{* * *}$ \\
\hline \multirow{2}{*}{ SAR(24) } & 0,2139 & 0,2147 \\
\hline & $(0,0398)^{* * *}$ & $(0,0312)^{* * *}$ \\
\hline \multirow{2}{*}{$\operatorname{SAR}(36)$} & 0,1773 & 0,1549 \\
\hline & $(0,0386)^{* * *}$ & $(0,0318)^{* * *}$ \\
\hline \multirow{2}{*}{$\sqrt{ } h_{t}$} & -- & $-0,0781$ \\
\hline & -- & $(0,0134)^{* * *}$ \\
\hline \multicolumn{3}{|c|}{ ECUACIÓN PARA LA VARIANZA CONDICIONAL } \\
\hline \multirow[t]{2}{*}{ C } & 0,0211 & 0,0005 \\
\hline & $(-0,0153)$ & $(-0,005)$ \\
\hline \multirow{2}{*}{$\varepsilon_{t-1}^{2}$} & 0,2194 & 0,1240 \\
\hline & $(0,0921)^{* * *}$ & $(0,0538)^{* * *}$ \\
\hline \multirow{2}{*}{$h_{t-1}$} & 0,7801 & 0,6516 \\
\hline & $(0,0521)^{* * *}$ & $(0,0615)^{* * *}$ \\
\hline \multirow{2}{*}{$\Pi_{t-1}$} & -- & 0,0805 \\
\hline & -- & $(0,0172)^{* * *}$ \\
\hline \multirow{2}{*}{ DUM } & $-0,1584$ & $-0,3600$ \\
\hline & $(-0,1043)$ & $(0,1821)^{* *}$ \\
\hline \multicolumn{3}{|c|}{ ESTADÍSTICOS DE DIAGNÓSTICO } \\
\hline $\begin{array}{l}\text { C. Estacionariedad: } \\
\qquad \alpha_{1}+\beta_{1}\end{array}$ & 0,9995 & 0,7756 \\
\hline R-Cuadrada & 0,817 & 0,8244 \\
\hline L-Likelihood & $-494,45$ & $-436,57$ \\
\hline $\mathrm{AIC}$ & 1,8718 & 1,6644 \\
\hline $\mathrm{BIC}$ & 1,9514 & 1,7599 \\
\hline D.W. & 1,6197 & 1,683 \\
\hline Obs & 539 & 539 \\
\hline
\end{tabular}

Nota: Los errores estándar se encuentran en paréntesis. * $0.10, * * 0.05$ y *** 0.01 .

Fuente: elaboración de los autores.

A continuación se desarrolla la prueba propuesta por Engle y $\mathrm{Ng}$ (1993), denominada prueba para sesgo de signo y tamaño, con la que se busca identificar la asimetría en la incertidumbre inflacionaria:

$$
\begin{gathered}
\varepsilon_{\mathrm{t}}^{2}=0,4392-0,2586 * \mathrm{~S}_{\mathrm{t}-1}^{-}-0,7693 \mathrm{~S}_{\mathrm{t}-1}^{-} * \hat{\varepsilon}_{\mathrm{t}-1}+ \\
0,9492 \mathrm{~S}_{\mathrm{t}-1}^{+} * \hat{\varepsilon}_{\mathrm{t}-1} \\
\mathrm{t}-\operatorname{stat}(2,1506)(-0,7865)(-1,9821)(4,5983)
\end{gathered}
$$


Dado que la hipótesis nula de no sesgo de signo y tamaño es $\mathrm{H}_{0}=\phi_{1}=\phi_{2}=\phi_{3}=0$, aunque $\phi_{1}=-0,2586$ no es estadísticamente significativo, el tamaño o magnitud de los choques $\phi_{2}=-0,7693$ y $\phi_{3}=0,9492$ sí presentan significancia estadística. La prueba conjunta calculando $\mathrm{N}^{*} \mathrm{R}^{2}$ de la regresión anterior, que asintóticamente seguirá una distribución $X^{2}$ con tres grados de libertad, es de 26,5528, con un $p$-value de 7.31E-06. Ello es evidencia de que choques positivos y negativos para $\hat{\varepsilon}_{\mathrm{t}-1}$ impactan de forma diferente sobre la varianza condicional y dominan los choques positivos. Con base en los resultados anteriores, se deben estimar modelos asimétricos no lineales para capturar de forma adecuada la incertidumbre inflacionaria, específicamente modelos GARCH-M, GJR-GARCH y E-GARCH.

\section{Modelo GJR-GARCH-M}

Una desventaja que presenta la estructura de un modelo GARCH es que supone simetría en la volatilidad de la inflación. El estimador $\varepsilon^{2}{ }_{t-1}$ del tercer y cuarto modelo captura los efectos sin distinguir la magnitud y el signo de estos; por tanto, si las buenas o malas noticias tienen diferentes efectos sobre la varianza condicional, y si $\chi_{\mathrm{i}} \neq 0$ se estará evidenciando la asimetría existente.

En la tabla 5 se presenta la estimación conjunta del modelo SARMA-GJR-GARCH(1,1)-M, las cuales indican que se cumplen las condiciones para la no negatividad del modelo GJR-GARCH mostrado en la tabla 2 , y que son $\alpha_{0}>, \alpha_{1}>0, \beta_{1} \geq 0$, y $\alpha_{1}+Y \geq 0$ (el modelo aún es admisible, incluso si $Y<0$ ). Se observa que el estimador correspondiente al componente del umbral $\left(\varepsilon_{t-1}^{2}{ }^{*} I_{t-1}\right)$ es negativo y estadísticamente significativo, y que $\alpha_{1}+Y_{1}=0,02$, lo que demuestra la condición de no negatividad y la presencia de efectos no simétricos de la inflación en la incertidumbre inflacionaria; esto es, que los choques ascendentes presentan un mayor efecto sobre la incertidumbre inflacionaria, tal como ya se había observado para la prueba de sesgo de signo y tamaño. Este efecto funciona en sentido inverso al apalancamiento, es decir, indica que los cambios en los precios tienden a correlacionarse positivamente con los cambios en la incertidumbre inflacionaria; por ejemplo, esto indicaría que un incremento en la variabilidad de los precios deriva en un aumento de su valor promedio.

Una vez capturado el efecto asimétrico, los resultados para la prueba de la hipótesis de

Tabla 5.

Resultados de las regresiones GJR-GARCH(1,1)-M (QML)

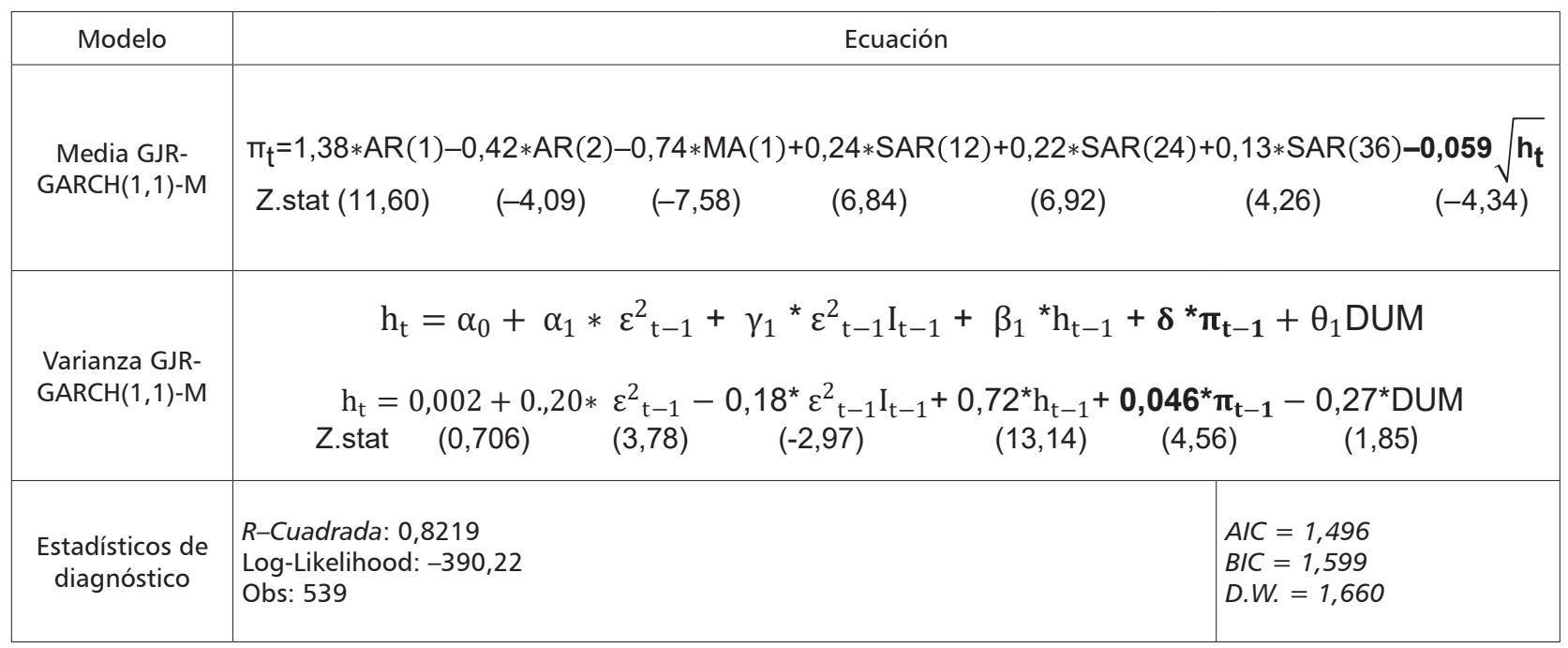

Fuente: elaboración de los autores. 
Friedman-Ball se siguen cumpliendo, es decir, que un incremento en la tasa de inflación provoca más incertidumbre inflacionaria; el valor preciso es de $(0,046)$, que es positivo y estadísticamente significativo. Respecto a la ecuación de la media, se observa que el error estándar condicional repercute de manera inversamente proporcional $(-0,059)$ sobre la inflación, lo que sostiene la hipótesis de un banco central estabilizador de Holland. Estos resultados son congruentes con los obtenidos por el segundo modelo tabla 4 , en la sección previa.

\section{Modelo E-GARCH-M}

Otro modelo capaz de capturar el efecto de asimetría existente es el desarrollado por Nelson (1991), también conocido como modelo E-GARCH, el cual permite expresar la varianza condicional $\left(h_{t}\right)$ en términos de logaritmos neperianos para presentar una forma funcional logarítmica lineal (log-lin).

En tabla 6 se presenta la calibración del modelo SARIMA-E-GARCH $(1,1)-M$; dado que la varianza condicional se expresa en logaritmos, no es necesario imponer restricciones artificiales de no negatividad sobre los parámetros. En este modelo en lugar de utilizar $\left(\varepsilon_{\mathrm{t}-1}^{2}\right)$ se utilizaron los residuos estandarizados para permitir una interpretación más natural del tamaño y persistencia de los choques; las estimaciones indican que el impacto de los choques sobre el logaritmo de la varianza condicional es $\alpha_{1}+\gamma_{1}=0,204+0,089=0,293$, lo que corrobora el efecto inverso al del apalancamiento mostrado en el modelo de la sección anterior.

Nuevamente el sistema de ecuaciones permite probar todas las hipótesis planteadas de forma simultánea. La segunda ecuación muestra, una vez más, evidencia estadística congruente con la hipótesis de Friedman y Ball, con un valor del coeficiente de la inflación rezagada un periodo de $(0,054)$, positivo y estadísticamente significativo; por su parte, en la primera ecuación se observa que la desviación estándar condicional está correlacionada negativamente con la inflación $(-0,050)$, además de ser estadísticamente significativa, lo cual estaría validando la hipótesis de un banco central estabilizador.

Tabla 6.

Resultados de las regresiones E-GARCH(1,1)-M (QML)

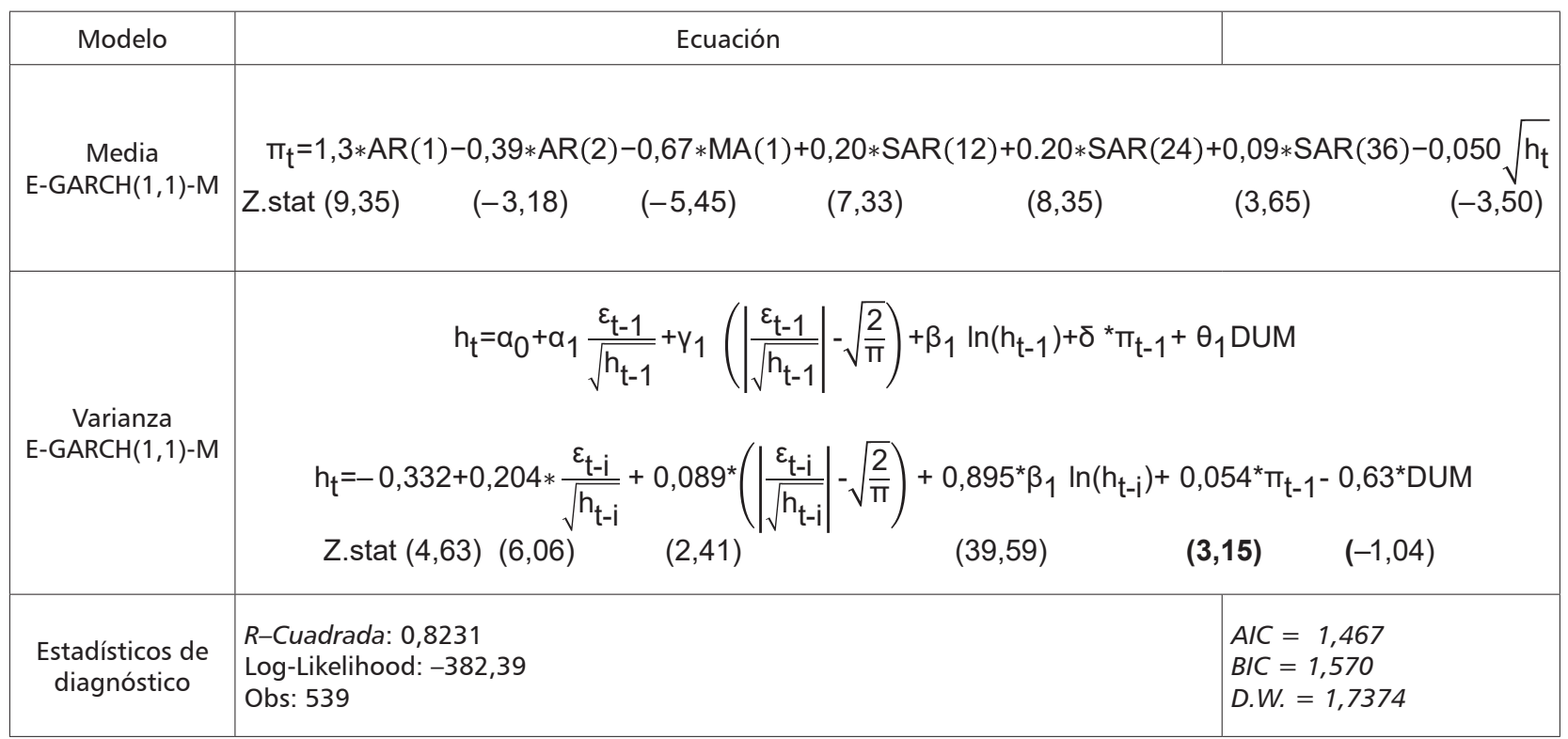

Fuente: elaboración de los autores. 


\section{CONCLUSIONES}

Los resultados sobre la relación entre el cambio porcentual de la inflación y la incertidumbre inflacionaria para el caso de la economía mexicana, utilizando datos mensuales sobre la inflación para el periodo enero 1969 a febrero 2017 , muestran que la función para la media se representa por componentes de tipo ordinario y estacional, es decir, se observa una fuerte presencia estacional en los datos. Asimismo, se comprueba que el cambio porcentual de la inflación se ve afectado por tres puntos de quiebre estructural: febrero de 1981, abril de 1988 y febrero de 1999.

El desarrollo de la metodología GARCH para medir la incertidumbre inflacionaria y de dos ecuaciones, una para la media adicionada por la desviación estándar condicional y la otra para la varianza condicional que se encuentra incrementada por la inflación rezagada un periodo y la variable de quiebres estructurales, arrojaron evidencia empírica que permite sostener tres conclusiones centrales. En primer lugar, para el periodo 1969-2017 se constata la presencia de una relación directamente proporcional que va del incremento de los precios hacia la volatilidad condicional (incertidumbre inflacionaria), lo que respalda la hipótesis de Friedman-Ball. Ello sugiere una postura fuertemente antiinflacionaria o estabilizadora del Banco de México, paradójicamente congruente con la visión acomodaticia del enfoque poskeynesiano; en otras palabras, esta posición ha resultado la más adecuada al instituto emisor para disminuir la incertidumbre sobre la inflación futura. Por tanto, tasas de inflación bajas y estables le han permitido a la autoridad monetaria reducir la incertidumbre inflacionaria. Es muy probable que en ciertos subperiodos, por ejemplo, durante la década de los 1980 y 1995-2001 (después de la crisis cambiariofinanciera y previamente a la adopción del esquema de metas de inflación) el Banco de México haya actuado de forma más oportunista que estabilizadora, en particular en años de elecciones (Stiglitz, 1998).

En segundo lugar, se comprueba la presencia de una relación causal negativa de la incertidumbre inflacionaria (nominal) sobre la inflación; esto significa que cuando la incertidumbre inflacionaria aumenta como resultado de un crecimiento de la inflación, Banxico responde contrayendo el crecimiento de la oferta monetaria para eliminar la incertidumbre. Este comportamiento es un indicador de que el Banco de México asume una postura de banco central estabilizador, la cual está asociada a un instituto central con mayor independencia en la ejecución y el diseño de sus políticas, es decir, se cumple la hipótesis de Holland.

Finalmente, se comprueba la presencia de un efecto asimétrico de los choques positivos sobre la tasa de cambio porcentual de la inflación. Utilizando una especificación de tipo GJR-GARCH-M y E-GARCH-M se infiere que son los choques positivos los que tienen una mayor incidencia sobre la varianza condicional de la inflación.

\section{REFERENCIAS}

1. Aguilar-Argaez A., Cuadra, G., Ramírez, C. y Sámano, D. (2014). Anclaje de las expectativas de inflación ante choques de oferta adversos. Ciudad de México: Banco de México.

2. Alesina, A. y Summers, L. (1993). Central bank independence and macroeconomic performance. Journal of Money, Credit, and Banking, 25, 151-162.

3. Baillie, R., Chung, C. y Tieslau, M. (1996). Analyzing inflation by the fractionally integrated ARFIMAGARCH model. Journal of Applied Econometrics, 11(1), 23-40.

4. Ball, L. (1992). Why does high inflation raise inflation uncertainty? Journal of Monetary Economics, 29(3), 371-388. 
5. Benati, L. y P. Surico (2008). Evolving US monetary policy and the decline of inflation predictability. Journal of the European Economic Association, 6, 634-646.

6. Berndt, E., Hall, B., Hall, R. y Hausman, J. (1974). Estimation and inference in nonlinear structural models. Annals of Economic and Social Measurement, 3, 653-665.

7. Bojanic, A. (2013). Inflación e incertidumbre inflacionaria en Bolivia. El Trimestre Económico, 80(2), 401-426.

8. Bollerslev, T. (1986). Generalized autoregressive conditional heteroskedasticity. Journal of Econometrics, $31(3), 307-328$.

9. Brooks, C. (2008). Introductory econometrics for finance. Cambridge: Cambridge University Press.

10. Broto, C. (2008). Inflation targeting in Latin America: empirical analysis using GARCH models. Madrid: Banco de España.

11. Caballero, C. y Caballero, R. (2015). Efectos asimétricos de schocks de inflación e incertidumbre inflacionaria en Bolivia 1990-2013. Economía Informa, 394, 68-99.

12. Capistran, C. and Ramos-Francia, M. (2006). Inflation dynamics in Latin America. Ciudad de México: Banco de México.

13. Caporale, G., Onorante, L. y Paesani, P. (2010). Inflation and inflation uncertainty in the Euro Area. Fráncfort: Banco Central Europeo.

14. Cogley, T., Primiceri G. y Sargent, T. (2009). Inflation-gap persistence in the US. Nueva York: Universidad de Nueva York.

15. Conrad, C. y Karanasos, M. (2005). On the inflation-uncertainty hypothesis in the USA, Japan and the UK: A dual long-memory approach. Japan and the World Economy, 17, 327-343.

16. Cukierman, A. (1992). Central Bank strategy, credibility, and independence. Cambridge: MIT Press.

17. Cukierman, A. y Meltzer, A. (1986). A theory of ambiguity, credibility and inflation under discretion and asymmetric information. Econometrica, 54(5), 1099-1128.

18. Cukierman, A., Webb, S. y Neyapti, B. (1992). Measuring th of Central Banks and its effect on policy outcomes. World Bank Economic Review, 6(3), 353-398.

19. Daal, E., Naka, A. y Sánchez, B. (2005). Re-examining inflation and inflation uncertainty in developed and emerging countries. Economics Letters, 89(2), 180-186.

20. Dincer, N. y Eichengreen, B. (2010). Central bank transparency: Causes, consequences and updates. Theoretical Inquiries in Law, 11(1), 75-123.

21. Enders, W. (2015). Applied Econometric Time Series. Nueva York: John Wiley.

22. Engle, R. and Ng, V. (1993). Measuring and testing the impact of news on volatility. Journal of Finance, 48(5), 1749-1778.

23. Fang W., Miller, S. y Yeh, C. (2010). Does a threshold inflation rate exist? Quantile inferences for inflation and its variability. Empirical economics, 39(3), 619-641.

24. Fountas, S. (2001). The relationship between inflation and inflation uncertainty in the UK: 1885-1998. Economics Letters, 74, 77-83.

25. Fountas, S. (2010). Inflation, inflation uncertainty and growth: Are they related? Economic Modelling, 27(5), 896-899. 
26. Fountas, S., Ioannidis, A. y Karanasos, M. (2004). Inflation, inflation uncertainty and a Common European monetary policy. The Manchester School, 72(2), 221-242.

27. Fountas, S., Karanasos, M. y Kim, J. (2006). Inflation uncertainty, output growth uncertainty, and macroeconomic performance. The Oxford Bulletin of Economics and Statistics, 68(3), 319-343.

28. Friedman, M. (1977). Nobel lecture: Inflation and unemployment. Journal of Political Economy, 85(3), 451-472.

29. Glosten, L., Jagannathan, R. y Runkle, D. (1993). On the relation between the expected value and the volatility of the nominal excess return on stocks. The Journal of Finance, 48(5), 1779-1801.

30. Golob, J. (1994). Does inflation uncertainty increases whit the inflation? Federal Reserve Bank of Kansas City Economic Review, 3, 2738.

31. Grier, R. y Grier, K. (2006). On the real effects of inflation and inflation uncertainty in México. Journal of Development Economics, 80(2), 478-500.

32. Grier, K. B. y Perry, M., (1998). On inflation and inflation uncertainty in the G-7 countries. Journal of International Money and Finance, 17(4), 671-689.

33. Grier, K. y Perry, M. (2000). The effects of real and nominal uncertainty on inflation and output growth: some GARCH-M evidence. Journal of Applied Econometrics, 15 (1), 45-58.

34. Grimme, C., Henzel, S. y Wieland, E. (2012). Inflation uncertainty revisited: Do different measures disagree? Múnich: Universidad de Múnich.

35. Holland, S. (1993a). Uncertain effects of money and the link between the inflation rate and inflation uncertainty. Economic Inquiry, 31, 39-51.

36. Holland, S. (1993b). Comment on 'inflation regimes and the sources of inflation uncertainty'. Journal of Money, Credit, and Banking, 25, 514-520.

37. Holland, S. (1995). Inflation and uncertainty: Tests of temporal ordering. Journal of Money, Credit and Banking, 27, 827-837

38. Hyndman, R. y Khandakar, Y. (2008). Automatic time series forecasting: The forecast package for r. Journal of Statistical Software, 27(3), 1-22.

39. Kontonikas, A. (2004). Inflation and inflation uncertainty in the United Kingdom: Evidence from GARCH modeling. Economic Modelling, 21 (3), 387-618.

40. McLeod, A. y Li, W. (1983). Diagnostic checking ARMA Time Series Models using Squared Residual Autocorrelations. Journal of Time Series Analysis, 4(4), 269-273.

41. Mántey, G. (2010). El "miedo a flotar" y la intervención esterilizada en el mercado de cambios como instrumento de la política monetaria en México. En G. Mántey y T. López (Coords.), Política monetaria con elevado traspaso del tipo de cambio. La experiencia mexicana con metas de inflación (pp. 165-196). Ciudad de México: Universidad Nacional Autónoma de México.

42. Nelson, D. (1991). Conditional heteroskedasticity in asset returns: A new approach. Econometrica, 59(2), 347-70.

43. Perrotini, H. y Rodríguez, D. (2012). Inflación, incertidumbre inflacionaria y crecimiento económico en México: 1929-2009. Revista Mexicana de Economía y Finanzas, 7(1), 1-26.

44. Polillo, S. y M., F. Guillen (2005). Globalization pressures: The global spread of central bank Independence. American Journal of Sociology, $110(6), 1764-1802$. 
45. Pourgerami, A. y Maskus, K. (1987). The effects of inflation on the predictability of price changes in Latin America: Some estimates and policy implications. World Development, 15(2), 287-290.

46. Press, W., Teukolsy, S., Vetterling, W. y Flannery, B. (1992). Numerical Recipes in Fortran. Cambridge: Cambridge University Press.

47. Ramos-Francia, M. y Torres, A. (2005). Reducción de la inflación a través del esquema de objetivos de inflación. La experiencia mexicana. Ciudad de México: Banco de México.

48. Rocabado, P (2009). Inflación e incertidumbre inflacionaria: un análisis para la economía boliviana 1937-2009 (tesis de grado). La Paz: Universidad Mayor de San Andrés.

49. Sidaoui, J. y Ramos-Francia, M. (2008). The Monetary Transmission Mechanism in Mexico: Recent Developments, BIS Papers. Recuperado de https://www.bis.org/publ/bppdf/bispap35q.pdf

50. Stiglitz J. (1998). Central banking in a democratic society. The Economist, 146(2), 196-226.

51. Thornton, J. (2007). The relationship between inflation and inflation uncertainty in emerging market economies. Southern Economic Journal, 73, 858-870.

52. Thornton, J. (2008) Inflation and inflation uncertainty in Argentina, 1810-2005. Economics Letters, 98(3), 247-252

53. Ungar, M. y B. Zilberfarb (1993). Inflation and its unpredictability - Theory and empirical evidence. Journal of Money, Credit, and Banking, 25(4), 709-720.

54. Zakoïan, J.M. (1994). Threshold heteroskedastic models. Journal of Economic Dynamics and Control, 18, 931-944.

55. Zeileis, A., Friedrich, L., Kurt, H. y Christian, K. (2002). Strucchange: An R package for testing for structural change in linear regression models. Journal of Statistical Software, Foundation for Open Access Statistics, 7(2). 